\title{
DESNUTRIÇÃO E INTERVENÇÃO NUTRICIONAL EM IDOSOS DE UMA INSTITUIÇÃO DE LONGA PERMANÊNCIA
}

\author{
MALNUTRITION AND NUTRITIONAL INTERVENTION IN \\ ELDERLY OF A LONG-STAY INSTITUTION
}

\author{
Silva, G. C. ${ }^{1}$, Mesquita, B. R. M. ${ }^{1}$, Benfica, M. E. ${ }^{1}$, França, V. F. ${ }^{1}$, Cardoso, L. G. V. ${ }^{1}$ \\ Afiliações: 1- Instituto Multiprofissional em Saúde - Campus Anísio Teixeira - Universidade Federal da Bahia. \\ Endereço do autor de correspondência: Rua Hormindo Barros, 58, Quadra 17, Lote 58. Bairro Candeias | CEP: 45.029-094 \\ | Vitória da Conquista - BA-Brasil. Email: luizgvc11@gmail.com.
}

\section{Resumo}

O envelhecimento populacional aumenta a demanda por Instituições de Longa Permanência (ILPI). Os idosos institucionalizados estão mais propensos ao risco nutricional ou desnutrição. Logo, é importante monitorar permanentemente as condições nutricionais, para implementar ações de intervenção nutricional. Objetivo do estudo foi avaliar a frequência de desnutrição em idosos institucionalizados, planejar, executar e avaliar os resultados de ações e estratégias de intervenção nutricional. Foram aferidas medidas antropométricas para estimar peso e altura, posteriormente, calcular e classificar pelo Índice de massa corporal (IMC) o estado nutricional. De forma complementar, foram usadas informações da circunferência da panturrilha $(C P)$ e adequação da circunferência do braço $(\% \mathrm{CB})$. As necessidades energéticas foram estimadas pela equação de bolso, seguida por intervenções nutricionais que priorizaram aumento do aporte energético e proteico nas refeições. Foram feitas análises descritivas e aplicado teste $T$ de Student em amostras pareadas para identificar mudanças no estado nutricional entre avaliação e reavaliação nutricional. Entre os 43 idosos a frequência de desnutrição foi de 60,5\% segundo IMC, 71,45\% para \% CB e 71,4\% para CP. Após as intervenções nutricionais 27 idosos foram reavaliados com a possibilidade de investigar o impacto das intervenções. As médias do peso $(50,74 \pm 11,10)$ e IMC $(21,40 \pm 4,36)$ na reavaliação foram superiores, porém não significativas em relação a avaliação inicial, $49,20 \pm 8,63$ e $20,75 \pm 3,13$, respectivamente $(p<0,05)$. $O$ estudo encontrou prevalência elevada de desnutrição, a intervenção nutricional resultou em aumento de algumas variáveis antropométricas, embora sem diferenças estatísticas, justificado pelo curto período da condução do monitoramento após intervenção.

Palavras-chave: Envelhecimento; Instituição de Longa Permanência para Idosos; Desnutrição; Intervenção nutricional.

\begin{abstract}
Population aging increases the demand for Long-Stay Institutions (LSI). Institutionalized elderly are more prone to nutritional risk or malnutrition. Therefore, it is important to permanently monitor nutritional conditions to implement nutritional intervention actions. The aim of the study was to assess the frequency of malnutrition in institutionalized elderly people, to plan, execute and evaluate the results of nutritional intervention actions and strategies. Anthropometric measures were taken to estimate weight and height, then calculate and classify the nutritional status by body mass index (BMI). In addition, information on calf circumference (CP) and adequacy of arm circumference (\% CB) were used. The energy needs were estimated by the pocket equation, followed by nutritional interventions that prioritized increased energy and protein intake in meals. Descriptive analyzes were performed and Student's $T$ test was applied to paired samples to identify changes in nutritional status between nutritional assessment and reassessment. Among the 43 elderly, the frequency of malnutrition was $60.5 \%$ according to BMI, $71.45 \%$ for\% $\mathrm{CB}$ and $71.4 \%$ for PC. After nutritional interventions, 27 elderly people were reevaluated with the possibility of investigating the impact of the interventions. The means of weight $(\mathbf{5 0 . 7 4} \pm 11.10)$ and BMI $(21.40 \pm 4.36)$ in the reassessment were higher, but not significant in relation to the initial assessment, $49.20 \pm 8.63$ and $20.75 \pm 3.13$, respectively $(p<0.05)$. The study found a high prevalence of malnutrition, nutritional intervention resulted in an increase in some anthropometric variables, although without statistical differences, justified by the short period of conducting monitoring after intervention.
\end{abstract}

Keywords: Aging; Long-stay institutions for the elderly; Malnutrition; Nutritional intervention. 


\section{Introdução}

A institucionalização dos idosos é emergente no Brasil e no mundo. Vem impulsionada pela necessidade do cuidado especializado no envelhecimento humano em especial para pessoas com algum grau de dependência e/ou com limitado suporte familiar (1). As mudanças na estrutura e na composição familiar, o aumento do número de mulheres que trabalham fora do lar e a redução dos arranjos familiares reduzem a atenção e a disponibilidade do cuidado com os idosos (2).

As mudanças fisiológicas e metabólicas normais do envelhecimento, tornam os idosos suscetíveis a desnutrição. (3) Este problema nutricional atinge 20 a $80 \%$ das pessoas residentes em Instituição de Longa Permanência a idosos - ILPI (4), prevalências estas, superiores comparados aos idosos que vivem na comunidade $(5,6)$. A etiologia da desnutrição é multifatorial, destacam-se as alterações sensoriais, edentulismo, inapetência, anorexia, disfagia, problemas digestivos e absortivos, déficit cognitivo, doenças crônicas e o uso de polifarmácia (5). O impacto negativo da institucionalização aos idosos ultrapassa as mudanças na rotina de vida, pode implicar em estresse, solidão, desgosto pela vida, redução da autoestima até a depressão (7) e também, provocar mudanças significativas na alimentação com repercussões na saúde global.

É reconhecido que o transcorrer do envelhecimento pode comprometer tanto a funcionalidade como a independência do idoso (6), resultando em fragilidade caraterizada pela Síndrome da Fragilidade (SF). Quando a SF está associada a desnutrição ampliam-se as limitações funcionais e os desfechos negativos em saúde (8-10), em especial esta condição é preocupante em idosos vulneráveis como é o caso daqueles institucionalizados. Um estudo (11) encontrou que a prevalência de SF foi maior $(51,5 \%)$ em idosos institucionalizados quando comparados aos idosos que vivem em comunidade $(16,2 \%)$.

As informações anteriores trazem à tona a problemática da desnutrição em idosos institucionalizados, porém, é incipiente o conhecimento sobre a realidade da condição nutricional das pessoas que vivem em ILPI do Sudoeste Baiano. Planejar e conduzir as ações de intervenção nutricional voltadas a superação dos problemas nutricionais em idosos 
institucionalizados foi uma das motivações para a realização do trabalho.

Diante do exposto, este estudo teve como objetivo avaliar a frequência de desnutrição em idosos institucionalizados, planejar, executar e avaliar os resultados de ações e estratégias de intervenção nutricional frente ao risco nutricional ou desnutrição.

\section{Métodos}

Estudo de intervenção nutricional em idosos de uma ILPI de um município do Sudoeste Baiano. Segundo o Atlas do Desenvolvimento Humano no Brasil (2010) o IDHM do município é de 0,678 , com destaque à dimensão da longevidade $(0,788)$. O município inclusive tem o IDHM superior ao estadual $(0,660)(12)$

A ILPI é uma instituição de natureza filantrópica e destinada a idosos independentes a dependentes de assistência à saúde interdisciplinar (13). Conta com a prestação de serviços de funcionários contratados, porém, é visto que boa parte do trabalho por profissionais de saúde é voluntário.

Amostra por conveniência, com intuito de incluir os 43 idosos institucionalizados entre janeiro a março de 2020. Foram coletadas dos prontuários informações sobre o sexo, idade, cor da pele, mobilidade, presença de disfagia ou problemas no funcionamento do Trato Gastrointestinal, diagnóstico de doenças e evolução, medicamentos e tratamentos de saúde, uso de suplementos nutricionais, disponibilidade de resultados de exames laboratoriais, prescrição da dieta e dietética, quando disponível. Além dessas informações, era mantido contato com a coordenadora do setor de enfermagem e cuidadores a fim de se conhecer o perfil individual dos idosos quanto ao estado de saúde, alimentação e nutrição.

Para avaliação antropométrica foram aferidas medidas de altura do joelho (AJ), circunferência da panturrilha (CP) e a circunferência do braço (CB), usadas para estimar o peso e a altura. As medidas foram aferidas conforme as técnicas propostas por Lohman (14), com fita métrica inextensível, com graduação de 1 mm, da marca MacroLife ${ }^{\circledR}$. Para a estimativa de peso $(\mathrm{kg})$ e a altura $(\mathrm{m})$ foram usadas as fórmulas matemáticas propostas por Chumlea, Guo, Roche (15) e Steinbaugh (16), respectivamente. A aferição das medidas foi executada na instituição, no ambulatório de enfermagem da ILPI, por discentes do curso de 
Nutrição com apoio dos técnicos de enfermagem da unidade. Foram realizadas duas avaliações antropométricas, a inicial e após dois meses após a intervenção nutricional, a fim de avaliar a efetividade dessa estratégia.

Para a avaliação nutricional foi utilizado o cálculo de índice de massa corporal, dado pela equação de peso $(\mathrm{Kg}) /$ altura $\left(\mathrm{m}^{2}\right)$. Para classificar o IMC foi usado os pontos de corte preconizados por Lipschitz (17). Para complementar o diagnóstico e o monitoramento nutricional foi considerado a $\mathrm{CP}$ e a adequação da CB. Para a adequação da CB foi realizado o cálculo com uso de percentis da National Health and Nutrition Examination Survey III (18). O ponto de corte para $\mathrm{CP}$ foram valores inferiores a $31 \mathrm{~cm} \mathrm{(19).}$

Após a avaliação nutricional as necessidades energéticas individualizadas foram estimadas pela equação de bolso considerando o estado nutricional. Para os idosos com desnutrição foi adotado os valores de 35 a 40 $\mathrm{Kcal} / \mathrm{Kg} /$ dia almejando a recuperação do estado nutricional. Já as necessidades proteicas foram baseadas na recomendação voltada a redução do risco de sarcopenia em idosos, que propõe 1,2 a 1,5 g de proteína/Kg/dia $(20,21)$. Já para os idosos desnutridos em catabolismo grave, foi considerado as recomendações da Sociedade Brasileira de Nutrição Parenteral e Enteral (BRASPEN) (22), que recomenda 1,5 a 2,0g de proteína $/ \mathrm{Kg} / \mathrm{dia}$.

Para os idosos com diagnóstico nutricional inicial de desnutrição ou em risco nutricional foi realizado o planejamento e a execução de intervenções nutricionais. As intervenções foram ajustadas as necessidades individuais e consideraram a consistência dos alimentos quando por via oral (normal, pastosa e disfágica), e mudança de dieta enteral artesanal para formulação enteral industrializada quando o idosos se encontravam em uso de sonda nasogástrica ou gastrostomia. A prescrição dietética considerou as doenças aguda ou crônica pré-existentes (hipertensão, diabetes, renal e câncer). Dietas especiais para diabetes priorizaram a substituição de produtos açucarados por sem açúcar, adição de fibras alimentares e ampliação do fracionamento das refeições, enquanto, para hipertensão o uso de controlado de sala no preparo de alimentos e substituição de temperos industrializados por naturais. Foi considerado a presença de alergias 
e intolerâncias alimentares e o funcionamento intestinal habitual.

Em linhas gerais as intervenções foram pautadas no aumento do aporte energético e proteico das refeições, com adoção de estratégias como a ampliação do número de refeições, a inclusão de alimentos de maior densidade energética disponíveis ou possíveis de aquisição na ILPI, tais como: acréscimo de fio de óleo de girassol, leite de coco, cereal às preparações (aveia), farinha de linhaça, oleaginosas triturada (amendoim), leite integral, sobremesas a base de frutas, açúcar mascavo, leite integral, entre outras preparações. Além disso, as intervenções voltaram-se a ampliação da oferta de alimentos fontes em proteínas, tais como ovos, leite, carnes e leguminosas e o uso de módulo proteico (albumina) acrescido às refeições, ou ainda, a complementação ou uso exclusivo da formulação enteral em pó para reconstituição destinada a idosos, com característica energética de $1,0 \mathrm{Kcal} / \mathrm{mL}$ e proteica de $0,36 \mathrm{~g} / \mathrm{mL}$. Quando necessário foram usados alimentos sem lactose, alimentos e preparações para diarreia (oferta de alimentos ricos em fibras solúveis, como maçã e goiaba, água de coco, suspensão da oferta de lactose) e alimentos para a constipação (aumento da oferta de verdura e folhosos e a oferta de suco laxativo).

Todas as propostas de intervenção nutricional foram encaminhadas para $o$ nutricionista voluntário do local, e após o consentimento, foram repassadas a pessoa responsável pela unidade de produção de alimentos que avaliou a viabilidade financeira e organizou a compra dos alimentos, módulos e suplementos nutricionais, otimizando o que tinha em estoque.

O projeto foi submetido e aprovado pelo Comitê de Ética em Pesquisa envolvendo seres humanos da Universidade Federal da Bahia (UFBA) campus Anísio Teixeira, pelo parecer número 4.190.431 e Certificado de Apresentação de Apreciação Ética (CAAE) número 32628920.2.0000.5556. O projeto dispensa o Termo de Consentimento Livre e Esclarecido (TCLE), pois tratam-se de idosos institucionalizados e sob responsabilidade do lar dos idosos, todavia, as aferições das medidas foram realizadas mediante o aceite voluntário do idoso.

Foi realizada análise descritiva, com os resultados expressos em média e desvio padrão. Para análise de comparação entre o as variáveis 
antropométricas entre a avaliação e reavaliação nutricional foi aplicado o teste de $T$ de Student para amostras pareadas. A efetividade da intervenção nutricional foi feita comparando-se as médias. As análises foram feitas com o software estatístico Statistical Package for the Social Sciences ${ }^{\circledR}$ (SPSS) versão 21 adotando o nível de significância de $95 \%$.

\section{Resultados}

Foram inicialmente avaliados e incluídos 43 idosos aptos a intervenção nutricional. Destas 43 pessoas, 16 não participaram da avaliação nutricional após a intervenção por não consentirem. A efetividade das estratégias de intervenção nutricional foi investigada a partir de informações de 27 idosos.

As características iniciais dos 43 participantes do estudo, estão descritas na tabela 1.

A média de idade da população avaliada inicialmente foi de 81,81 \pm 10,2 anos (mínimo de 60 e máximo de 102 anos). A maioria dos idosos institucionalizados eram do sexo feminino, de raça/cor branca e cadeirantes. Dentre as doenças crônicas avaliadas, foi possível verificar que entre os 43 idosos, 21 possuíam hipertensão arterial sistêmica (HAS), enquanto apenas 15 eram portadores de Diabetes Mellitus (DM). Também foi observado que a minoria dos idosos eram disfágicos, e faziam o uso de polifarmácia.

Em relação ao estado nutricional houve predomínio da desnutrição segundo o IMC (60,5\%), adequação da CB $(72,1 \%)$ e a CP $(71,4 \%$,$) , porém, a duas últimas foram mais$ sensíveis para predizer a desnutrição (Tabela 1).

Quanto as ações de intervenção nutricional, todos os idosos que inicialmente encontravam-se com risco nutricional ou desnutrição tiveram ampliação da oferta energética e proteína, seja, exclusivamente com ajustes da alimentação ou suplementação. Foi realizada adaptações de consistência da dieta para $16 \%$ dos idosos, substituição do uso de dieta enteral artesanal pela formulação enteral industrializada para 2,3\% dos idosos com acesso nasogástrico. Foram realizados ajustes na alimentação e adoção de dietas especiais para $34,9 \%$ das pessoas diabéticas e 48,8\% dos hipertensos. Considerando o funcionamento do TGI foi feita prescrição de dieta laxativa para $22,2 \%$. Além disso, para 2,3\% dos idosos foram feitas prescrições específicas devido a presença de intolerância à lactose. 
Tabela 1. Caracterização inicial dos idosos institucionalizados no Sudeste da Bahia.

\begin{tabular}{|c|c|c|}
\hline Variáveis & $\mathbf{N}$ & Porcentagem (\%) \\
\hline \multicolumn{3}{|l|}{ Sexo } \\
\hline Feminino & 35 & 81,4 \\
\hline Masculino & 8 & 18,6 \\
\hline \multicolumn{3}{|l|}{ Raça } \\
\hline Branca & 40 & 93 \\
\hline Preta & 3 & 7 \\
\hline \multicolumn{3}{|l|}{ Mobilidade } \\
\hline Deambula & 13 & 30,2 \\
\hline Deambula com ajuda & 3 & 7,0 \\
\hline Cadeirante & 27 & 62,8 \\
\hline \multicolumn{3}{|l|}{ Disfagia } \\
\hline Sim & 9 & 16,1 \\
\hline Não & 34 & 60,7 \\
\hline \multicolumn{3}{|l|}{ HAS } \\
\hline Sim & 21 & 48,8 \\
\hline Não & 22 & 51,2 \\
\hline \multicolumn{3}{|l|}{ DM } \\
\hline Sim & 15 & 34,9 \\
\hline Não & 28 & 65,1 \\
\hline \multicolumn{3}{|l|}{ Polifarmácia } \\
\hline Sim & 13 & 30,2 \\
\hline Não & 30 & 69,8 \\
\hline \multicolumn{3}{|l|}{ Suplementos nutricionais } \\
\hline Não faz uso & 40 & 93 \\
\hline Faz uso & 3 & 7 \\
\hline \multicolumn{3}{|l|}{ IMC } \\
\hline Desnutrição & 26 & 60,5 \\
\hline Eutrofia & 13 & 30,2 \\
\hline Sobrepeso & 4 & 9,3 \\
\hline \multicolumn{3}{|l|}{$\% \mathrm{CB}$} \\
\hline $\begin{array}{l}\text { Desnutrição leve, moderada } \\
\text { ou grave }\end{array}$ & 31 & 72,1 \\
\hline Eutrofia & 9 & 20,9 \\
\hline Sobrepeso ou obesidade & 3 & 7,0 \\
\hline \multicolumn{3}{|l|}{$\mathbf{C P}$} \\
\hline Desnutrição & 30 & 71,4 \\
\hline Eutrofia & 12 & 28,6 \\
\hline
\end{tabular}

Fonte: ILPI de Vitória da Conquista - Ba (2020). 
A tabela 2 apresenta as médias das variáveis antropométricas antes e depois das intervenções nutricionais. Foi possível perceber que houve aumento das variáveis peso, IMC e $\% \mathrm{CB}$.

Tabela 2. Médias para as variáveis antropométricas de idosos institucionalizados antes e após a intervenção nutricional.

\begin{tabular}{lccccc}
\hline \multirow{2}{*}{ Variáveis } & N & \multicolumn{2}{c}{ Avaliação inicial } & \multicolumn{2}{c}{ Avaliação após intervenção } \\
\cline { 3 - 6 } & & Média & DP & Média & DP \\
\hline Peso $(\mathbf{K g})$ & 27 & 49,1737 & 8,62526 & 50,7419 & 11,09197 \\
IMC $\left(\mathbf{K g} / \mathbf{m}^{2}\right)$ & 26 & 20,7419 & 3,13276 & 21,3804 & 4,35661 \\
CP $(\mathbf{c m})$ & 26 & 28,1846 & 3,70488 & 28,1692 & 3,75497 \\
CB $(\boldsymbol{\%})$ & 27 & 100,0996 & 13,49534 & 102,0048 & 15,39002 \\
\hline Fonte: ILPI de Vitória da Conquista - Ba (2020)
\end{tabular}

Fonte: ILPI de Vitória da Conquista - Ba (2020).

A tabela 3 traz a comparação das variáveis antropométricas antes e após as intervenções nutricionais. Segundo análise, não foi verificado significância estatística entre as variáveis antes e após ações de intervenção nutricional.

Tabela 3. Comparação das variáveis antropométricas de idosos institucionalizados antes e após a intervenção nutricional.

\begin{tabular}{lccccc}
\hline \multicolumn{1}{c}{ Variáveis } & Média & DP & \multicolumn{2}{c}{ IC 95\% da diferença } & p-valor \\
\hline Peso $(\mathbf{K g})$ & 1,56815 & 5,66019 &,- 67095 & 3,80725 & 0,162 \\
IMC $\left(\mathbf{K g} / \mathbf{m}^{2}\right)$ &, 63846 & 2,49932 &,- 37104 & 1,64796 & 0,205 \\
CB $(\boldsymbol{\%})$ & 1,90519 & 6,96595 &,- 85045 & 4,66082 & 0,167 \\
CP $(\mathbf{c m})$ &,- 01538 & 1,09460 &,- 45750 &, 42673 & 0,943 \\
\hline Fonte: Teste - T de Student para amostras pareadas.
\end{tabular}

\section{Discussão}

O predomínio de mulheres corrobora com os achados de um estudo em duas ILPI no Município de Fortaleza (23), e reafirma a maior expectativa de vida para as mulheres (24). Com referência a raça, o resultado foi semelhante a outro estudo (25) e reflete as características gerais da população brasileira, na qual a maioria se autodeclara branca (26). No que concerne a mobilidade dos idosos, houve o predomínio de 
cadeirantes com limitações de funcionalidade.

Resultados similares foram encontrados por Trennepohl, Hansen e Thum (27) com idosos institucionalizados da cidade de Cruz Alta/RS.

A respeito da presença de doenças crônicas entre os idosos institucionalizados, os resultados obtidos, apontam, a maior prevalência de portadores de HAS em relação a DM, o que vai de encontro com outras pesquisas $(28,29)$.

Apesar dos resultados do presente estudo demostrarem uma minoria de idosos disfágicos, a literatura mostra grande associação da disfagia e a desnutrição, com repercussões na funcionalidade e na capacidade física (10).

Em relação à polifarmácia, os dados do presente estudo mostraram que os idosos que faziam a prática de polifarmácia também eram minoria. Divergindo de tal resultado, várias pesquisas $(28,29)$ demostram que é comum o predomínio da polifarmácia em idosos que residem em ILPI. Assim como sua associação com a desnutrição (10).

A frequência de desnutrição nesse estudo com idosos institucionalizados foi elevada, corroborando com prevalências descritas por outros autores $(4,5,6)$. Além disso, os efeitos da desnutrição são especialmente mais dramáticos em idosos institucionalizados.

A prevalência de desnutrição em idosos institucionalizados é realidade no Brasil, porém, com diferenças por regiões. Schmidt et al (30) conduziu uma pesquisa em ILPI no interior do Rio Grande do Sul e, verificou elevada prevalência de desnutrição $(34,1 \%)$ pelo IMC. No Rio Grande do Norte, Andrade et al (25) encontrou frequência elevada $(89,4 \%)$ para desnutrição em idosos institucionalizados.

É sabido que a presença de múltiplas doenças crônicas, o uso de polifarmácia e a redução da capacidade funcional ou dependência funcional são fatores que podem alterar a ingestão alimentar e provocar desequilíbrio nutricional em idosos. Adicionalmente, as mudanças de hábitos e rotinas impostas para os idosos institucionalizados podem potencializar as alterações nutricionais a estes indivíduos (8).

Para a avaliação do estado nutricional o IMC é o indicador antropométrico mais utilizado, contudo, para idosos o uso exclusivo tem limitações como a validade das medidas de peso e altura (31). O uso de medidas complementares de CB e CP são recomendadas (32). Os resultados encontrados permitem dizer 
que as medidas de $\mathrm{CP}$ e adequação da $\mathrm{CB}$ foram mais sensíveis para o diagnóstico de desnutrição do que o IMC. A maior sensibilidade da medida de CP foi verificada em outro estudo (33) com 30 idosas residentes de uma ILPI da cidade de Recife/PE. Da amostra avaliada 40\% apresentou estado nutricional inadequado pela $\mathrm{CP}$, enquanto para o IMC a frequência foi de $16,7 \%$ de desnutrição. Em outro estudo (34) foi encontrado maior sensibilidade para identificar idosos com desnutrição com a adequação da $\mathrm{CB}$ em relação ao IMC

É sabido que o estado nutricional é um importante marcador das condições de saúde no idoso. Seu reconhecimento é imprescindível para promover a intervenção nutricional (31). No estudo em questão, o perfil nutricional inicial foi crucial para planejar estratégias nutricionais, respeitando as individualidades.

A fim de prevenir e controlar a desnutrição em idosos, diferentes estratégias de intervenção nutricional são reconhecidas e recomendadas, que vão, desde o ajuste no cardápio e adaptações das refeições até o uso de suplementos nutricionais, para a melhora do equilíbrio nutricional (35).
As intervenções nutricionais adotas foram pautadas no uso de suplemento proteico (22g de proteína por porção) e na formulação enteral (11g de proteína por porção), adicionados em vitaminas ou em mingaus, nos lanches da manhã, tarde e na ceia. Este planejamento foi feito com base nas necessidades energéticas/ proteicas individuais estimadas. Para além dos suplementos, foi feita a inclusão de alimentos de maior densidade energética e proteica, como o acréscimo de fio de óleo de girassol e ovos nas refeições. Foram revistas e adequadas as consistências dos alimentos e preparações condicionados as condições fisiológicas do envelhecimento e a presença de doenças.

Na ILPI em questão, as refeições são majoritariamente preparadas com alimentos oriundos de doações, sendo essa a realidade para a maioria das entidades filantrópicas. Mesmo com as limitações para planejar e viabilizar as adequações das refeições dos cardápios, destacase a preocupação e o esforço da ILPI em conseguir doações e/ou comprar com recursos próprios, em especial para os módulos proteicos e fórmula enteral especializada para as demandas do envelhecimento. 
Nas observações feitas durante as refeições mostraram que os idosos quando quisessem poderiam repetir as refeições, e suas preferências alimentares eram respeitadas. Os idosos que necessitavam de auxílio para se alimentar recebiam atenção dos cuidadores, contudo, devido ao restrito número de cuidadores, as refeições eram rapidamente ofertadas. Observou-se que para os idosos que conseguiam alimentar-se sozinhos, estes não recebiam estímulo, e muitas vezes, não se alimentavam satisfatoriamente.

É notório que o trabalho do cuidador é complexo e cansativo. É importante que estes profissionais sejam treinados permanentemente e sejam vigilantes e colaborativos as necessidades individualizadas dos idosos, almejando o estímulo a alimentação e redução do risco nutricional.

Ao comparar as variáveis antes e depois das intervenções nutricionais, foi verificado mudanças positivas nas médias do peso, IMC e \%CB, embora sem significância estatística. Porém, a ausência de associação não refuta a importância das intervenções nutricionais para a melhora do estado nutricional dos idosos, uma vez que, foi claro a mudança positiva nas médias e na melhora nutricional gradual dos participantes. Em vista disso, é relevante divulgar o impacto de intervenções nutricionais planejadas e executadas em ILPIS, mesmo frente as limitações existentes.

Em uma meta-análise feita por Poscia et al (36) foi visto que apesar dos amplos estudos feitos a respeito das intervenções nutricionais, ainda se sabe muito pouco sobre sua real eficácia. Ainda assim, a suplementação oral, isto é, uso de produtos à base de proteínas ou complexos vitamínicos, mostrou ser a forma de intervenção mais efetiva, onde $50 \%$ dos artigos estudados demonstraram bons resultados na ingestão alimentar e calórica, no ganho de peso, na diminuição do risco de má nutrição e na prevenção de quedas e fraturas.

Entre as limitações do estudo destacamse a impossibilidade da continuidade do monitoramento nutricional frente as intervenções nutricionais almejadas, devido a instalação da pandemia por Covid-19 e a necessidade de manter o isolamento social. Outra limitação, foi a dificuldade do acesso as informações atualizadas em prontuários, o que dificultou a compreensão do histórico de saúde e determinar a frequência da Síndrome da 
Fragilidade, bem como a condução de

intervenções mais complexas. Como pontos fortes, destaca-se o delineamento do estudo de intervenção nutricional em idosos institucionalizados e a preocupação em estudar e sanar a lacuna do conhecimento sobre a temática nesta população com características peculiares. Por fim, a pesquisa possibilitou mostrar a importância do olhar especializado do nutricionista na equipe interdisciplinar para atuar em prol da superação da vulnerabilidade nutricional de idosos institucionalizados.

\section{Conclusão}

Após a análise do perfil nutricional dos idosos, foi encontrado elevada prevalência da desnutrição, independente das variáveis antropométricas usadas para o diagnóstico.

Ao avaliar o impacto da intervenção nutricional nas variáveis antropométricas e estado nutricional dos idosos, verificou-se aumento das médias, porém não significativo. É possível vislumbrar que a intervenção nutricional continuamente poderia trazer relevância estatística, contudo, nossos achados não refutam a importância clínica dessas ações à saúde dos idosos que participaram da intervenção.
O estudo reafirma a importância da atenção especializada da equipe interdisciplinar em saúde para a superação da vulnerabilidade nutricional dos idosos institucionalizados.

\section{Referências}

1. Fagundes KVDL, Esteves MR, Ribeiro JHM, Siepiersk CT, Silva JV, Mendes MA. Instituições de longa permanência como alternativa no acolhimento das pessoas idosas. Rev. Salud Pública. 2017; 19 (2): 210-214.

2. Alves MB, Menezes MR, Felzemburg RDM, Silva VA, Amaral JB. Instituições de longa permanência para idosos: aspectos físicoestruturais e organizacionais. Rev Enferm. 2017; 21(4).

3. Oliveira KDL, Haack A, Fortes RC. Terapia nutricional na lesão por pressão: revisão sistemática. Rev. bras. geriatr. Gerontologia 2017;.20 (4): 567-575.

4. Mendes GM, Oliveira TC. Assistência Nutricional em Instituição de Longa Permanência para Idosos: Relato de Experiência. Rev. Bras. de Educação e Cultura. 2018; 156165.

5. Bermejo RV, Garcia IA, Galera DM, Las Heras Rodriguez M, Torramade JP. Prevalencia de desnutrición en personas mayores institucionalizadas em España: un análisis multicéntrico nacional. Rev Nutr Hosp. 2015; 31(3):1205-1216.

6. Damo CC, Doring M, Alves ALS, Portella MR. Risco de desnutrição e os fatores associados em idosos Institucionalizados. Rev. Bras. Geriatr. Gerontol. 2018; 21(6): 735-742.

7. Magagnin T, Santiago GDC, Dutra JDDR, Silva SD, Nunes RZS, Rabelo JS, et al. O Olhar Multiprofissional em uma Instituição de Longa Permanência para Idosos de Santa Catarina. Rev. Mult. Psic. 2019; 13 (45): 591-593. 
8. Silva LD, Silva NC, Ferreira ES, Castro LC, Alencar MSS. Fatores de risco que potencializam fragilidades em idosos institucionalizados. Research, Society and Development. 2020; 9 (5)

9. Mesquita AF, Silva EC, Eickemberg M, Roriz AKC, Barreto - Medeiros JM, Ramos LB. Factors associated with sarcopenia in institutionalized elderly. Nutr. Hosp. 2017; 34 (2): $345-351$

10. Moreira NCF, Krausch-Hofmann S, Matthys C, Vereecken C, Vanhauwaert E, Declercq A, et al. Risk factors for malnutrition in Older Adults: A 18 systematic Review of the Literature Based on Longitudinal Data. Adv Nutr. 2016; 7 (3):507-22.

11. Panes, VCB. Qualidade de vida e síndrome da fragilidade em idosos [Tese de Doutorado]. Bauru - SP: Faculdade de Odontologia de Bauru; 2017.

12. Programa das Nações Unidas para o Desenvolvimento Instituto de Pesquisa Econômica Aplicada [homepage na internet]. Atlas de Desenvolvimento Humano no Brasil. 2010. [acesso em 20 de abr de 2020]. Disponível em: http:atlasbrasil.org.br.

13. Conselho Nacional do Ministério Público. Manual de atuação funcional: o Ministério Público na Fiscalização das Instituições de Longa Permanência para Idosos. Brasília: Conselho Nacional do Ministério Público, 2016.

14. Lohman TG, Roache AF, Martorell R. Anthropometric standardization reference manual. Illinois: Human Kinetics Books. 1988.

15. Chumlea WC, Guo S, Roche AF, Steinbaugh ML. Prediction of body weight for the nonambulatory elde rly from anthropometry. J Am Diet Assoc. 1988; 88: 564-568.

16. Chumlea WC, Roche AF, Steinbaugh ML. Estimating stature from knee height for persons 60 to 90 years age. J Am Geriatr Soc. 1985; 33 (2): 116-120.

17. Lipschitz DA. Screening for nutritional status in the elderly. Primary Care - Clinics in Office Practice. 1994; 21 (1): 55-67.
18. National Institutes of Health, National Heart Lung and Blood Institute. Clinical guidelines on the identification, evaluation, and treatment of overweight and obesity in adults. In: National Institutes of Health National Heart Lung and Blood Institute, editors, 1998.

19. World Health Organization. Obesity: preventing and ma naging the global epidemic. Geneva; 1998. [WHO - Technical Report Series]

20. Paddon-Jones D, Short KR, Campbell WW, Volpi E, Wolfe RR. Role of dietary protein in the sarcopenia of aging. Am J Clin Nutr 2008; 87(5): 1462S-6S.

21. Waters DL, Baumgartner RN, Garry PJ, Vellas B. Advantages of dietary, exerciserelated, nad therapeutic interventions to prevent and treat sarcopenia in adult patients: na update. Clin Interv Aging 2010; 5: 259-70.

22. Gonzalez MC, Horie LM, Campos ACL, Waitzberg DL, Nascimento JEAS, Correia MITD et al. Braspen Journal. São Paulo. 2018; $33(8)$.

23. Lima APM, Gomes KVL, Pereira FGF, Barros LM, Silva MG da, Frota NM. Avaliação nutricional de idosos residentes em instituições de longa permanência. Rev. baiana enferm. 2017; 31(4)

24. Egydio L. Do Feminismo à Feminização: Gênero e Envelhecimento em uma sociedade em transformação. Rev Portal de Divulgação. 2017; (54)

25. Andrade FLJP, Lima JMR, Fidelis KNM, Jerez-Roig J, Lima KC. Incapacidade cognitiva e fatores associados em idosos institucionalizados em Natal, RN, Brasil. Rev. Bras. Geriatr. Gerontol (online). 2017; 20 (2): 186-197

26. Instituto Brasileiro de Geografia e Estatística [homepage na internet]. Censo 2010: Características da população e dos domicílios [acesso em 27 abr 2020]. Disponível em: https://biblioteca.ibge.gov.br/visualizacao/perio dicos/93/cd_2010_caracteristicas_populacao_d omicilios.pdf

27. Trennepohl C, Hansen D, Thum C. Características de saúde de idosos institucionalizados. In: XXII Seminário 
Interinstitucional de Ensino, Pesquisa e Extensão; Rio Grande do Sul: Universidade de Cruz Alta; 2017.

28. Gatto CM. Caracterização das condições de saúde e presença de polifarmácia em idosos institucionalizados [Dissertação de pósgraduação]. Passo Fundo - RS: Universidade de Passo Fundo; 2017.

29. Reis KMC, Jesus CAC. Relationship of polypharmacy and polypathology with falls among institutionalized elderly. Texto Contexto Enferm, 2017; 26(2): e3040015

30. Schmidt L, Soder TF, Volkweis DSH, Benetti F. Avaliação nutricional de idosos institucionalizados de uma ILPI do interior do estado do Rio Grande do Sul. RBCEH. 2017; 14 (1): 83-92.

31. Santos ANPC, Silva IB, Oliveira J. Perfil alimentar e nutricional de idosos residentes em instituição de longa permanência [Trabalho de Conclusão de Curso]. Araçatuba - SP: Centro Universitário Toledo; 2016.

32. Wijnhoven

HAH ,Schueren MAEB , Heymans MW, De Vet

HC, Kruizenga HM, Twisk JM, et al. Low MidUpper Arm Circumference, Calf Circumference, and Body Mass Index and Mortality in Older Persons. J Gerontol A Biol Sci Med Sci. 2010; 65 (10): 1107-1114.

33. Gottschall C,Tarnowski M, Machado P, Raupp D, Marcadenti, Rabito EL, A et al. Predictive and concurrent validity of the Malnutrition Universal Screening Tool using mid-upper arm circumference instead of body mass index. J Hum Nutr Diet. 2019; 32, 775780.

com recursos do Plano Nacional de Assistência Estudantil - PNAES, Decreto no. 7.234 de 19/07/2010.
34. Nogueira GMB, Oliveira Neto J, Carlos DMO. Avaliação nutricional de idosos dependentes funcionais institucionalizados: uma visão antropométrica. Revista Varia Scientia Ciências da Saúde. 2017; 3 (1).

35. Paino Pardal L, Poblet i Montells L, Ríos Álvarezc L. Mayores que viven solos $\mathrm{y}$ malnutrición. Rev Atencion Primaria. 2017; 49(8):450 - 458.

36. Poscia A, Milovanovic S, La Milia DI, Duplaga M, Grysztar M, Landi F, et al. Effectiveness of nutritional interventions addressed to elderly persons: umbrella systematic review with meta-analysis. Eur J of Public Health. 2018; 28 (2): 275-283.

Agradecimentos - Gostaríamos de agradecer à todos que contribuíram direta e indiretamente na construção desse projeto e ao programa Sankofa pelo apoio financeiro para realização dessa pesquisa.

Financiamento - Bolsista do Programa Sankofa, edição 2018/2019. O Programa Sankofa é uma iniciativa da Coordenação de Ações Afirmativas, Educação e Diversidade CAAED da Pró Reitoria de Ações Afirmativas e Assistência Estudantil (PROAE), mantido Declaração de conflitos de interesse - Nós autores declaramos que não há conflitos de interesse.

Reservado aos Editores

Data de submissão: 23/11/2020

Data de aprovação: 02/12/2020 